\title{
Method for enhancing the resolution of holographic displays
}

\author{
S. B. Hasan, ${ }^{1}$ T. Kozacki ${ }^{2}$ \\ ${ }^{1}$ Institute of Condensed Matter Theory and Solid State Optics, FSU Jena, Germany. \\ ${ }^{2}$ Institute of Micromechanics and Photonics, Warsaw University of Technology, ul. Św. A. Boboli 8, 02-525 Warsaw, \\ Poland.
}

Received December 7, 2009; accepted December 24, 2009; published December 31, 2009

\begin{abstract}
We present an investigation into the resolution limit and its enhancement for holographic display systems implemented by a liquid crystal on silicon spatial light modulators (SLMs). The problem of resolution of the displayed objects is connected with a limited pixel pitch of the spatial light modulator. Furthermore, it delimits the angular view-ability of reconstructed objects, which is the most important factor influencing the applicability of display systems. In this regard, we will present a solution using multiple SLMs set to work together. Such a configuration allows us to ease the resolution limit which in turn provides larger angular view-ability. A theoretical analysis based on a plane wave spectrum is presented, followed by numerical simulations, to demonstrate the utility of the concept.
\end{abstract}

In holographic displays [1], the extent of angular viewability remains one of the most important factors affecting the experience of watching reconstructed holographic objects. It is delimited by the finite pixel pitch of the particular SLM device used to implement holographic data. Recently, several attempts have been made to enhance this feature by employing innovative optical modules in their designs. In one approach, electrical or optical spatial light modulators are aligned such as to generate a large number of data points [2]. In another, viewing space perspectives are individually identified which are generated by individual SLMs whose normal vectors point to the scene of view [3]. However, it provides improved resolution in a particular direction at the cost of reduced resolution in another by the same factor as an increase in resolution. While it might be a practical answer in some circumstances, a method which can enhance the angular view-ability in any particular direction(s) without compromising the same in others can also come in handy in a variety of cases. This can include situations when either the pitch of an SLM device used is already so large that a further decrease in resolution in any particular direction may significantly deteriorate the viewing quality. Therefore, in this letter we present a new method which is theoretically rigorous and provides a continuous plane wave spectrum, much like as if a technologically enhanced system were employed. We put to work a configuration of two SLMs which results in an optical field of the object having the bandwidth twice as that produced by a system based on a single SLM of the same technology without losing the spatial extent of the optical field.
The interdependence of angular view-ability and resolution of the SLM device can be understood by visiting the plane wave spectrum formulation of diffracted optical field given by [4]:

$$
\begin{gathered}
U(\boldsymbol{r})= \\
\int_{-\infty}^{\infty} \int_{-\infty}^{\infty} A\left(f_{x}, f_{y}\right) \mathrm{e}^{i k_{z} z} \mathrm{e}^{i 2 \pi\left(f_{x} x+f_{y} y\right)} d f_{x} d f_{y} \\
k_{z}=\sqrt{\left(4 \pi^{2} / \lambda^{2}-\left(f_{x}^{2}+f_{y}^{2}\right)\right)} \\
A\left(f_{x}, f_{y}\right)=F . T[U(x, y, 0)]
\end{gathered}
$$

According to the Nyquist criterion, in the case of a discretized optical field, which is the SLM plane, the maximum frequency in either horizontal or vertical direction is equal to half the sampling frequency. Combining it with eq. (1) leads us to the important result relating directional view-ability with the pixel pitch of an SLM. Without any loss of generality, in the case of normal incidence of an input reconstruction beam, the maximum diffraction angle is given by:

$$
\theta_{\max }=\arcsin \left(\frac{\lambda}{2 \Delta}\right)
$$

According to eq. (4), the smaller the pixel pitch $\Delta$, the larger the value of $\theta_{\max }$ in the given direction. And the larger the value of $\theta_{\max }$ in a direction, the greater the angular view-ability. Therefore, the problem of increasing angular view-ability is, in fact, a problem of improving the resolution of an SLM device and vice versa. With these two aspects of the problem in mind, we present in the following section a novel technique which can help us achieve greater angular view-ability and therefore resolution. Phase holograms will be used as a conceptual device to conceive practical explanations and numerical implementation of our methods, where necessary and possible. The reason for preferring phase holograms over others is the superior diffraction efficiency they provide over other counterparts.

Our solution is based on the insight we achieve from Eq. (1) and (4), and it allows us to continuously distribute 
the plane wave spectrum generated by each SLM. According to Eq. (4), the effect of limited resolution of the SLM device manifests in the form of a limit on the maximum angle of inclination of plane waves emanating out of the diffracting plane. To emulate an SLM with higher resolution and which can therefore produce plane waves with higher inclination angles, we place multiple SLMs of a given resolution that are tilted with respect to each other and encode different bands of object's spatial frequency spectrum into each of them. The idea is illustrated in Fig. 1, which shows the case of two SLM devices placed to emulate an SLM with twice as high resolution as the two constituent devices. The major effect of inclination is to introduce a d.c. shift in the plane waves emanating out of respective devices when viewed with respect to the image plane.

The mathematical computation of the angle $\theta$ which we need to tilt each SLM device will now follow. To keep computations simple, we will present the case in which we increase the bandwidth in only one direction, say $\mathrm{x}$ axis. The effect of computing an electromagnetic field on a frame of reference tilted with respect to the input plane is the introduction of a shift in the spatial frequency spectrum accompanied by shape distortion which can increase or decrease the size of the frequency spectrum depending upon the angle of inclination $\theta$. As Fig.1 shows, we use one SLM to cover the positive half of the reconstructed object's frequency band while the other SLM (is what?) for the negative.

Let us take $f_{x}$ as the horizontal spatial frequency on the plane parallel to the tilted SLM and $f_{x}{ }^{\prime}$ as the same frequency component on the tilted plane. The transformation can then be mathematically written as $[5$, 6]:

$$
\begin{gathered}
f_{x}=f_{x} \cos \varphi_{1}-\sqrt{\lambda^{-2}-\left(f_{x}^{2}+f_{y}^{2}\right)} \sin \varphi_{1} \\
A s f_{x, \min }=-\frac{1}{2 \Delta_{x}} \rightarrow f_{x}=0 \\
=0=-\frac{1}{2 \Delta_{x}} \cos \varphi_{1}-\sqrt{\lambda^{-2}-\left(f_{x}^{2}+f_{y}^{2}\right)} \sin \varphi_{1} \\
\varphi_{1}=-\arctan \left(\frac{1}{\sqrt{\left(2 \Delta_{x} / \lambda\right)^{2}-\left(1+\left(2 \Delta_{x} f_{y}\right)^{2}\right)}}\right)
\end{gathered}
$$

where $\phi_{1}$ is the angle by which the SLM is tilted with respect to the object plane. We can repeat the same analysis for the other SLM covering the negative half of frequency spectrum to find out that $\phi_{1}=-\phi_{2}$. This result is expected due to the symmetry of the problem.
After knowing the tilt angle required for making two SLMs work, we now have to determine the total object's frequency which we can access by this method. It is not straightforward because as mentioned before, one effect of tilting the image plane is the change in the size of a frequency band. In order to find out this $\Delta f_{x}{ }^{\prime}$ contributed by the single SLM, we once again make use of Eq. (5), as follows:

$$
\begin{gathered}
\Delta f_{x}^{\prime}=f_{x, \max }^{\prime}-f_{x, \min }^{\prime} \\
f_{x, \min }^{\prime}=0 \\
f_{x, \max }^{\prime}=f_{x, \max } \cos \varphi-w \sin \varphi \\
f_{x, \max }^{\prime}=\frac{1}{2 \Delta_{x}} \cos \varphi-\sqrt{\lambda^{-2}-\left(\frac{1}{4 \Delta_{x}^{2}}+f_{y}^{2}\right)} \sin \varphi
\end{gathered}
$$

By repeating the same analysis for the second SLM, it can be shown that in the present configuration of two SLMs, $f_{x, \min }^{\prime}=-f_{x, \max }^{\prime}$. Therefore, $\Delta f_{x}{ }^{\prime}=2 f_{x, \max }^{\prime}$ is the total spatial bandwidth of the reconstructed object along x-axis while the resolution $\Delta_{x}{ }^{\prime}=1 /\left(2 f_{x, \max }^{\prime}\right)$.

Optically, we can implement the aforementioned idea as schematically shown in Fig. 2. A plane wave illuminates the two tilted SLMs through a beam splitter which then combines with the reflection of the two SLMs in to a continuous wave output having the spatial frequency extent double the size of that produced by original SLM devices.

In order to computationally simulate the theoretically presented solution above, we employ a computer generated hologram obtained using an iterative algorithm [7]. We modified the algorithm to adapt it for our setup into the form described in two major steps as follows:

a) Restricting the optical field amplitude at hologram and hologram reconstruction planes.

b) Back and forth propagation of optical fields.

As in our case we have two holograms and one reconstructed object, we have to employ a constant amplitude constraint at both hologram planes. During back propagation, we propagate the whole extended optical field from reconstruction plane to hologram. Since this field now has to be assigned to two and not one SLM which is normally the case, we divide the frequency spectrum into two and assign it to the two tilted SLMs. The forward propagation i.e. from hologram plane to reconstruction plane is carried out exactly in the reverse manner. 


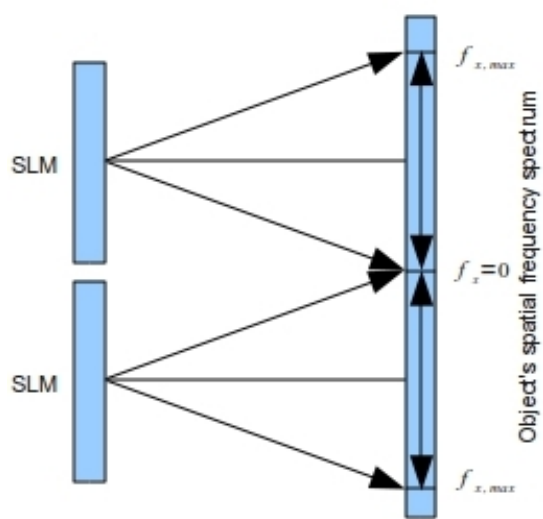

Fig. 1. Selection of object's frequency spectrum for each SLM.

Using the aforementioned iterative algorithm, we generated a test object with the frequency spectrum composed of spatially distributed frequency patches at $0.1190,0.1044,0.0898,0.0751,0.0605$, and $0.0459 \mu \mathrm{m}^{-1}$. The pixel size was assumed to be $8 \mu \mathrm{m}$ and therefore the Nyquist frequency equal to $0.0625 \mu^{-1}$. This is chosen with the view of keeping correspondence with commercially available liquid crystals to facilitate later verification of computational results through an experiment.

With these parameters, we performed a numerical simulation of the setup shown in Fig. 2 to obtain optical reconstruction of the object. Fig. 3a shows the Fourier spectrum of the reconstructed intensity image through two SLMs while Fig. $3 \mathrm{~b}$ shows, for the purpose of comparison, the frequency spectrum obtained through SLM1 only. It is clear that we achieved an extended frequency spectrum twice the size of the Nyquist frequency limit by two SLMs configuration of Fig. 2, twice as much as what is technologically possible through a single device alone.

The method presented in sec. 3 is exact, as noted earlier. It requires somewhat rigorous computation for determining the tilt angle given to each SLM as well as demanding the full frequency spectrum of the object in enhanced resolution to be known beforehand for obtaining optical profile of holograms used. Therefore, this method is suitable if higher frequency contents of an object are needed with best possible accuracy.

We have presented a method whereby the resolution and hence the angular view-ability of holographic systems based on discretized spatial light modulators could be increased. The resultant holographic image has a continuous spatial distribution of optical field and so does a continuous spatial frequency spectrum. Another advantage of this method over the known ones is the possibility to enhance resolution in any direction without having to loose the same in any other direction.

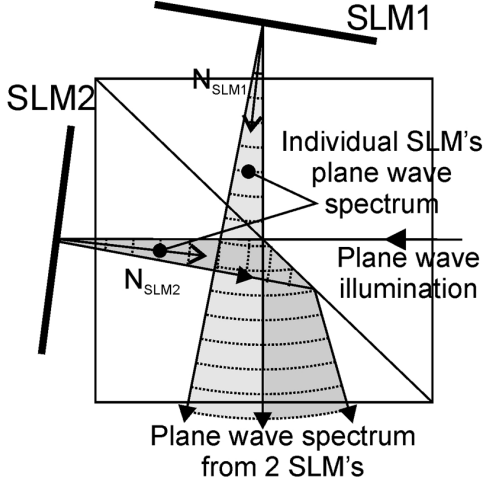

Fig. 2. Schematic diagram of the setup showing two tilted SLMs configured to generate a combined output.
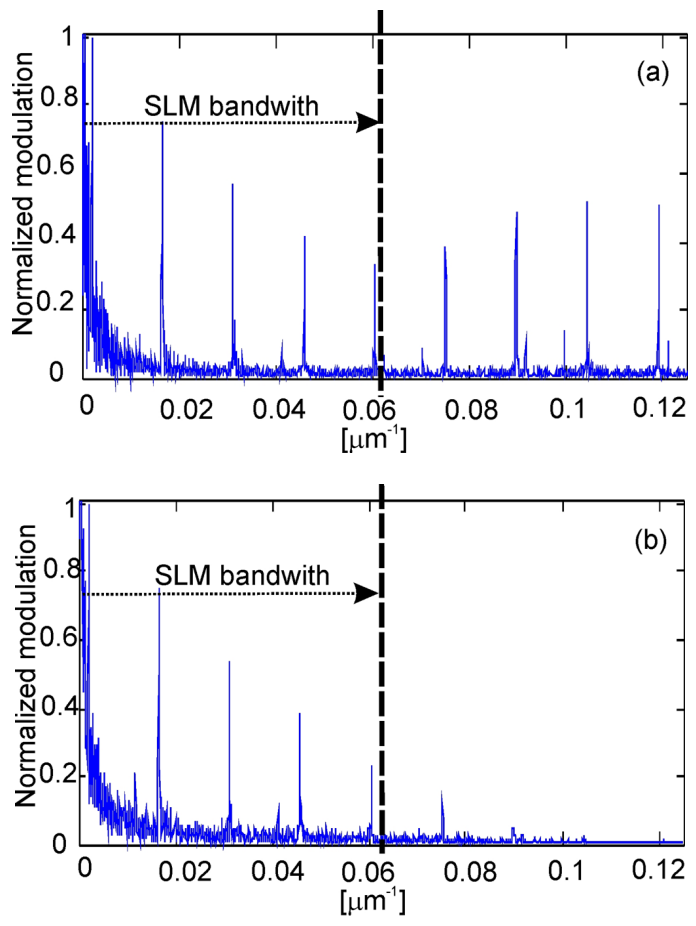

Fig. 3. (a) Frequency spectrum of intensity pattern of the reconstructed holographic image generated by two SLMs. (b) Frequency spectrum produced by single SLM.

\section{References:}

[1] P. Benzie, J. Watson, P. Surman, I. Rakkolainen, K. Hopf, H. Urey, V. Sainov, and C. von Kopylow, IEEE Trans. Circuits Syst. Video Technol. 17, 1647-1658 (2007).

[2] K. Maeno, N. Fukaya, O. Nishikawa, K. Sato, and T. Honda, Proc. SPIE 2652, 15-23 (1996).

[3] J. Hahn, H., Y. Lim, G. Park, B. Lee, Opt. Express 16, 12372-12386 (2008).

[4] J. W. Goodman, "Introduction to Fourier Optics", 3rd ed. (Roberts \& Company Publishers, 2004).

[5] N. Delen and B. Hooker, J. Opt. Soc. Am. A 15, 857-867 (1998).

[6] K. Matsushima, H. Schimmel, and F. Wyrowski, J. Opt. Soc. Am. A 20, 1755-1762 (2003).

[7] J. R. Fienup, Appl. Opt. 21(15), pp. 2758-2769, 1982. 